OPEN ACCESS

Edited by:

Joe Kossowsky,

Boston Children's Hospital and

Harvard Medical School,

United States

Reviewed by:

Jay R. Malone,

Washington University School of Medicine in St. Louis, United States

Sarah Goff

University of Massachusetts Amherst,

United States

${ }^{*}$ Correspondence:

Dhurgshaarna Shanmugavadivel shaarnashan@doctors.org.uk

Specialty section

This article was submitted to General Pediatrics and Pediatric

Emergency Care,

a section of the journal

Frontiers in Pediatrics

Received: 16 December 2021 Accepted: 02 February 2022

Published: 25 February 2022

Citation:

Buonsenso D, LiU J-F

Shanmugavadivel $D$, Davis $T$ and Roland D (2022) Impact of Diversity in

Training Resources on

Self-Confidence in Diagnosing Skin Conditions Across a Range of Skin

Tones: An International Survey.

Front. Pediatr. 10:837552.

doi: 10.3389/fped.2022.837552

\section{Impact of Diversity in Training Resources on Self-Confidence in Diagnosing Skin Conditions Across a Range of Skin Tones: An International Survey}

\author{
Danilo Buonsenso 1,2,3, Jo-Fen Liu ${ }^{4}$, Dhurgshaarna Shanmugavadivel ${ }^{4 *}$, Tessa Davis ${ }^{5,6}$ and \\ Damian Roland ${ }^{7,8}$ on behalf of Don't Forget the Bubbles
}

${ }^{1}$ Department of Woman and Child Health and Public Health, Fondazione Policlinico Universitario A. Gemelli IRCCS, Rome, Italy, ${ }^{2}$ Dipartimento di Scienze Biotecnologiche di Base, Cliniche Intensivologiche e Perioperatorie, Università Cattolica del Sacro Cuore, Rome, Italy, ${ }^{3}$ Global Health Research Institute, Istituto di Igiene, Università Cattolica del Sacro Cuore, Rome, Italy, ${ }^{4}$ Academic Unit of Population and Lifespan Science, University of Nottingham, Nottingham, United Kingdom, ${ }^{5}$ Paediatric Emergency Department, Royal London Hospital, London, United Kingdom, ${ }^{6}$ Blizard Institute, Queen Mary University of London, London, United Kingdom, ' Paediatric Emergency Medicine Leicester Academic Group, Children's Emergency Department, Leicester Royal Infirmary, Leicester, United Kingdom, ${ }^{8}$ SAPPHIRE Group, Health Sciences, Leicester University, Leicester, United Kingdom

Background: Medical images are invaluable in facilitating recognition of clinical signs. Recent studies highlight a lack of diversity of skin tone images used within medical education. However, there is a paucity of data on the impact of this on patient care.

Aims: To investigate diversity in training resources used by users of an International online teaching platform and self-confidence in diagnosing skin conditions in all skin tones.

Methods: Users of an online teaching platform (www.dftbskindeep.com) were invited to participate in a survey evaluating key points including geographical location, ethnicity, profession, specialty, years of experience, training resources and confidence in diagnosing skin conditions. Data analyses were performed using SPSS. Categorical variables were presented as proportions. Chi-squared or Fisher's exact tests were used to compare the distribution between groups as appropriate.

Results: Of 600 participants, $74 \%$ reported training resources featuring predominantly white skin. Participants were "generally uncertain" in $43 \%$ cases, "sometimes uncertain but clinically safe" (52\%), and "confident across a range of skin tones" in a minority (5\%). Self-confidence was associated with location [higher in Africa (29\%) and Latin America (11\%), $(p<0.001)$ ]; diversity of training resources [higher with a mix (10\%) or darker tones $(20 \%)(p<0.001)$ ]; clinical experience [6-10 (5\%) or $>10$ years of practice $(11 \%)(p<0.001)$ ] and specialty [highest in dermatologists (53\%, $p<0.001)$ ]. Self-confidence was lowest among pediatricians, emergency medicine and pediatric emergency medicine specialists $(<5 \%)$. 
Conclusions: These data provide preliminary evidence that training resources used by healthcare professionals on a global scale may lack enough diversity on representation of skin images, and a lack of self-confidence in diagnosing pediatric skin conditions. Further work is needed to understand the impact on knowledge and patient care to ensure equitable healthcare for all.

Keywords: child health, pediatrics, medical education, dermatology, ethics, diverity, skin tones

\section{INTRODUCTION}

Race, a recognized social determinant of health, has a profound impact on the health status of children, adolescents, emerging adults, and their families ${ }^{1},{ }^{2}$. Although significant progress has been made in the last decade, there is still clear evidence of the negative impact of one's race on their health and wellbeing, through implicit and explicit biases, institutional structures, and interpersonal relationships (1). Unconsciously, the modern health system is contributing to feeding structural racism in healthcare. An example is demonstrated by skin color. Studies assessing diversity of skin representation among textbooks in the United States (US), Canada $(2,3)$ and in major scientific journals (4-7) have shown that the majority of images used within these educational materials are of light skin tones (defined in the oxford English dictionary as the color of the surface of someone's skin). Furthermore, descriptors of dermatological features also lack diversity; many descriptions of rashes focus on redness, pallor, purpura and cyanosis all of which are more difficult to recognize in darker skin tones or may not be present at all. Inequalities extend from education to research with evidence that children with darker skin tones have a lower probability of inclusion in clinical trials (8). The COVID-19 pandemic further highlighted these inequalities. Whilst evidence shows that Afro-Carribean and Asian patients experience a more severe illness course from COVID-19 (9), a systematic literature review demonstrated that articles describing the cutaneous manifestations of COVID-19 almost exclusively used clinical images from patients with light skin tones, with no single published images of darker skin tones (Fitzpatrick type V or VI) (10). This has the potential implication that patients, including children, with darker skin tones are more likely to experience a delayed or incorrect diagnosis compared to their lighter skin tone counterparts. Importantly, in a study assessing Afro-Caribbean patients' perception of their dermatology care, specialized knowledge in assessment and treatment of darker skin tones and hair was recognized by patients as a contributing factor for better care satisfaction (11).

The impact of the lack of representation in medical resources on the ability of healthcare professionals to recognize childhood skin conditions, however, has never been clearly documented. This is despite the American Academy of Pediatrics clearly advocating for rigorous research that examines, amongst others, "the impact of policy changes and community-level interventions on reducing the health effects of racism and other forms of

\footnotetext{
${ }^{1}$ https://www.nejm.org/race-and-medicine

${ }^{2}$ https://www.thelancet.com/racial-equality
}

discrimination on youth development" (1). This represents a large gap in the literature that needs to be urgently addressed.

This study aims to add to the dearth of data in this area by performing a pilot investigation of self-declared diversity of medical training resources used by healthcare professionals using an online teaching platform, and self-confidence in diagnosing skin conditions on different skin tones globally.

\section{METHODS}

An online platform was developed (www.dftbskindeep.com) hosting visual and teaching resources of dermatological conditions on a wide range of skin tones. The core team comprises international experts in the field of pediatric emergency medicine, pediatric infectious diseases, and pediatric dermatology, working in both Europe and Low-Middle Income Countries (LMICs).

The platform has been widely shared on social media and those who accessed the website were able to register and participate.

Participants were asked to $\log$ in and self-declare the following information (details on the information required and language are available on the registration page of the website; https:// dftbskindeep.com/register/): continent of practice, ethnicity, profession, specialty, years of experience, training resources used within training (white skin, a mix of skin tones, darker skin tones only), and self-confidence in diagnosing skin conditions (generally uncertain if correct, sometimes uncertain but clinically safe, confident across range of skin tones). For this study purpose, responses collected between 26/08/2020 and 07/01/2021 were included. Since the aim of this pilot investigation is to assess the self-declared need of resources, and training materials, including a wider range of skin tones, we did not include a detailed classification of skin tones. Participants were made aware that non-identifiable demographic information would be shared as part of a research project prior to declaring the information. Given the methodology and the voluntary participation in the survey through a free website, ethical approvals were not deemed necessary. In the United Kingdom, where this study was conducted, the National Institute for Health Research (NIHR) deem health care professional research of this kind as not requiring formal ethical review (hra-decisiontools.org.uk).

\section{Statistical Analyses}

Given the absence of previous data, a target sample size was set at 500 participants, in keeping with similar self-assessment and 
evaluative questionnaires run for Pediatric Acute Care topics of investigation $(12,13)$.

Data analyses were performed using IBM SPSS 26.0 for Windows (IBM Corp, Armonk, NY, USA). Categorical variables were presented as proportions, and Chi-squared or Fisher's exact tests were used to compare the distribution between groups as appropriate. A $p$-value of $<0.05$ was deemed statistically significant in all analyses.

The Chi-Square Automatic Interaction Detector (CHAID) was used to further explore the interactions between respondent's characteristics and their confidence level. The dependent variable in the model was self-reported confidence (root node), and independent variables included participant's geographical location, ethnicity, experience, profession, specialty and majority training resources. At each step, the algorithm determines the best next split based on the magnitude of Bonferooni-adjusted $\chi^{2}$ statistics. Variable sub-categories are merged if they are not statistically different with respect to the outcome variable. The significance level for splitting nodes or merging categories was set at 0.05 (14). The variable that shows strongest relationships with the dependent variable appears in the first node of the decision tree. Node formation and segment configuration continue iteratively until no further splits can be performed, or the sample size in parent/child nodes is $<30$.

\section{RESULTS}

\section{Study Population}

During the study period, 2,316 website visitors accessed the quiz registration page and 615 participants $(26.5 \%)$ completed the survey. Fourteen were excluded for duplicate or incomplete data, one because the specialty reported was not eligible. Six hundred participants were included in the final analysis (Table 1). Participants responded from all continents, although Europe (56\%) and Oceania (23\%) were the most represented. The majority of participants were white/caucasian (69\%). Four hundred and thirty-nine (73\%) were clinicians, mostly pediatricians $(37 \%)$, emergency doctors $(22 \%)$ pediatric emergency doctors (12\%) and a minority were dermatologists (3\%). Just over half $(56 \%)$ reported at least 6 years of practice since qualification.

Participants were asked to report if the majority of training resources used during their training were inclusive of materials with a range of skin tones. Four hundred and forty-one (74\%) reported that only white skin was usually represented, while resources including a mix of skin tones $(24 \%)$ or other skin tones (3\%) were less represented. The diversity of training resources used across continents and according to ethnicity are shown in Supplementary Table 1. In Latin America and Africa, resources with only white skin images were reported in 44 and $29 \%$ respondents, respectively.

\section{Self-Confidence in Diagnoses of Skin Conditions Across Different Skin Tones}

Overall, $43 \%$ of participants reported that they are "generally uncertain if correct", $52 \%$ were "sometimes uncertain but
TABLE 1 | Characteristics of the survey respondents $(n=600)$.

\begin{tabular}{|c|c|c|}
\hline & $n$ & Col\% \\
\hline \multicolumn{3}{|l|}{ Continent } \\
\hline Europe & 337 & $56 \%$ \\
\hline Oceania & 140 & $23 \%$ \\
\hline America & 59 & $10 \%$ \\
\hline Latin America & 9 & $2 \%$ \\
\hline Asia & 41 & $7 \%$ \\
\hline Africa & 14 & $2 \%$ \\
\hline \multicolumn{3}{|l|}{ Ethnicity } \\
\hline White & 411 & $69 \%$ \\
\hline Asian/Oriental & 124 & $21 \%$ \\
\hline Black or African & 29 & $5 \%$ \\
\hline Hispanic or Latino & 10 & $2 \%$ \\
\hline Unclassified & 26 & $4 \%$ \\
\hline \multicolumn{3}{|l|}{ Majority training resources } \\
\hline White skin & 441 & $74 \%$ \\
\hline A mix of skin tones & 144 & $24 \%$ \\
\hline Darker skin tones & 15 & $3 \%$ \\
\hline \multicolumn{3}{|l|}{ Experience } \\
\hline Student & 58 & $10 \%$ \\
\hline $1-2$ years & 65 & $11 \%$ \\
\hline $3-5$ years & 129 & $22 \%$ \\
\hline $6-10$ years & 188 & $31 \%$ \\
\hline 11 years + & 159 & $27 \%$ \\
\hline \multicolumn{3}{|l|}{ Profession } \\
\hline Medic* & 439 & $73 \%$ \\
\hline Advanced nursing practitioner & 47 & $8 \%$ \\
\hline Nursing & 60 & $10 \%$ \\
\hline Paramedic and Other $\mathrm{HCP}^{\dagger}$ & 21 & $4 \%$ \\
\hline Other (not specified) & 33 & $6 \%$ \\
\hline \multicolumn{3}{|l|}{ Specialty } \\
\hline Emergency medicine & 129 & $22 \%$ \\
\hline Emergency paediatrics & 69 & $12 \%$ \\
\hline Primary care & 93 & $16 \%$ \\
\hline Paediatrics & 224 & $37 \%$ \\
\hline Dermatology & 17 & $3 \%$ \\
\hline Other (not specified) & 68 & $11 \%$ \\
\hline \multicolumn{3}{|l|}{ Confidence in diagnoses } \\
\hline Generally uncertain if correct & 259 & $43 \%$ \\
\hline Sometimes uncertain but clinically safe & 309 & $52 \%$ \\
\hline Confident across range of skin tones & 32 & $5 \%$ \\
\hline
\end{tabular}

*Including medical doctors/primary care practitioner (all grades) and medical student ( $n=$ 3), physician associate or assistant (4) and physician assistant-student (1).

t' Including paramedic (16) and clinical pharmacist (1), pharmacist (1), physiotherapist (1), podiatrist (1) and respiratory therapist (1).

clinically safe", with only $5 \%$ "confident across a range of skin tones".

Table 2 and Supplementary Figures 1,2 show the correlations between demographics and reported self-confidence in diagnosing skin conditions across different skin tones. Self-confidence level was associated with geographical location, 
TABLE 2 | Self-reported confidence in diagnosis.

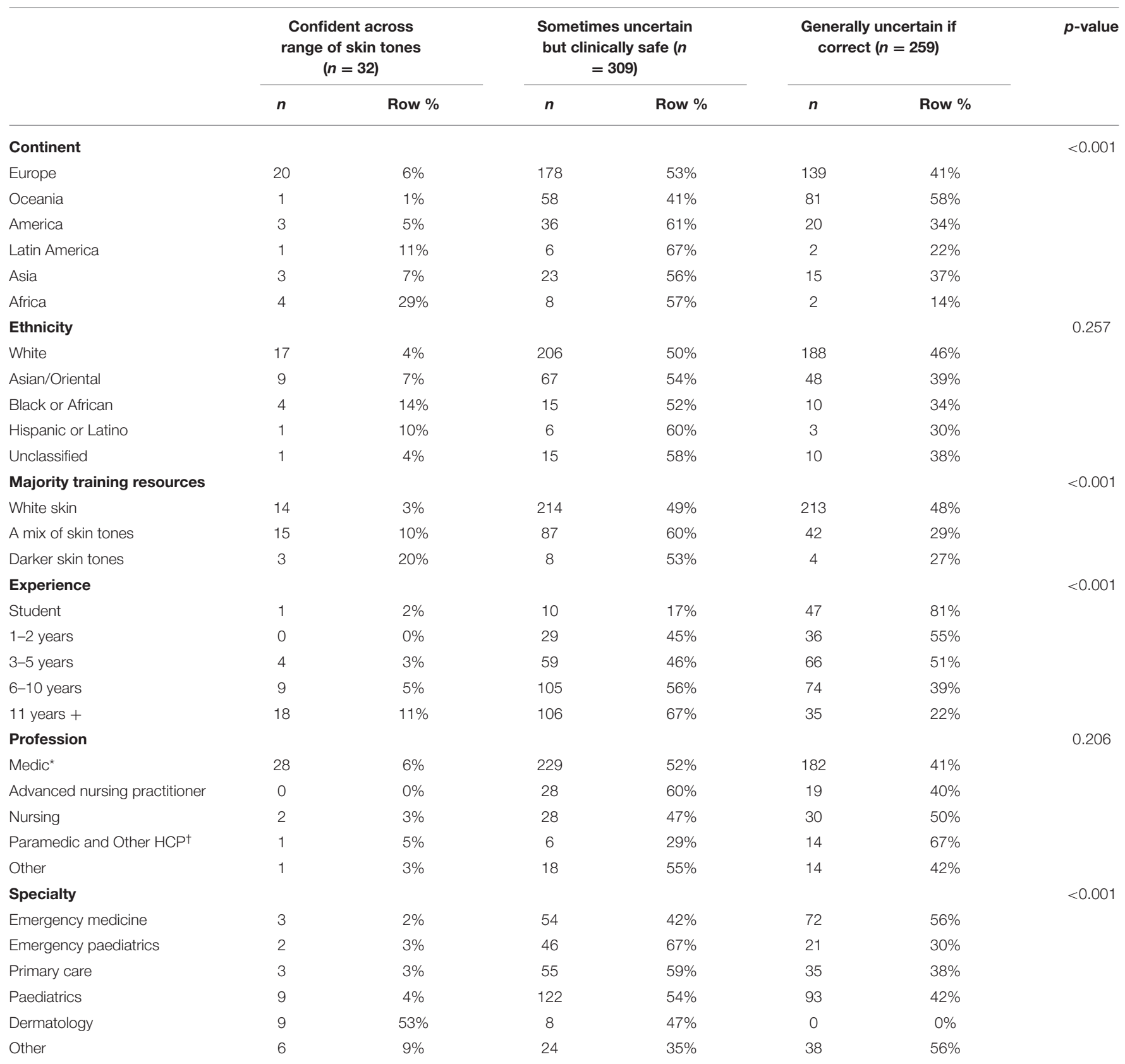

*Including medical doctors/primary care practitioner (all grades) and medical student ( $n=3)$, physician associate or assistant (4) and physician assistant-student (1).

tIncluding paramedic (16) and clinical pharmacist (1), pharmacist (1), physiotherapist (1), podiatrist (1) and respiratory therapist (1).

diversity of training resources used, specialty and years of experience. Those living in Africa (4/14, 29\%) and Latin America $(1 / 9,11 \%)$ were most confident across a range of skin tones which was statistically significant $(p<0.001)$. Confidence was higher in those who trained using a mix of skin tones $(15 / 144,10 \%)$ or darker skin tones only $(3 / 15,20 \%)(p<0.001)$. Confidence was also highest in those with more clinical experience [6-10 (9/188, $5 \%)$ or $>10$ years of practice $(18 / 159,11 \%)(p<0.001)]$, and by specialty [highest in dermatologists $(9 / 17,53 \%, p<0.001)$ ].
Self-confidence was very low among pediatricians, emergency medicine and pediatric emergency medicine specialists $(<5 \%)$. Ethnicity did not seem to affect self-confidence ( $p>0.05)$.

Figure 1 and Supplementary Table 2 explore the relationships between self-reported confidence in diagnosing skin conditions across different skin tones and other factors. Years of clinical experience was the main parameter positively affecting self-confidence, with those having at least 10 years of experience being the most self-confident $(p<0.0001)$. The 


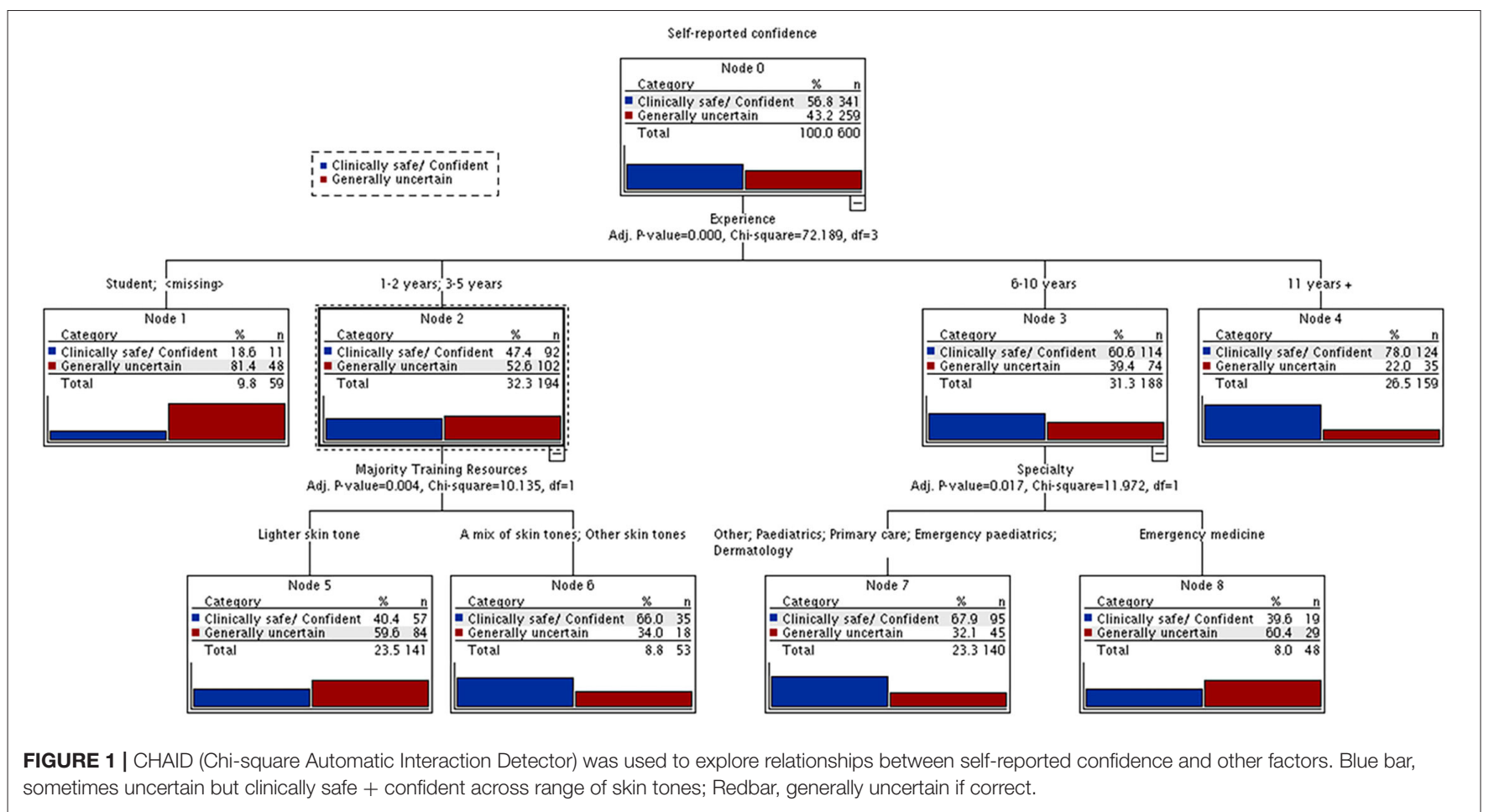

impact of training resources was also analyzed in those groups whose self-confidence according to years of experience was borderline. Those with 1-5 years of experience were generally uncertain in $52.6 \%$ of cases. The type of resources used during training significantly affected self-confidence, with those who reported a mix of skin tones or exclusively darker skin tone resources being significantly more self-confident $(p=0.004)$. Furthermore, by specialty, those working in emergency medicine were least confident $(p=0.01)$.

\section{DISCUSSION}

In this study, we asked users of an international teaching online platform to self-declare the diversity in training resources used during their education and their self-confidence in diagnosing skin conditions in a range of skin tones. To our knowledge, this is the first international study to answer this crucial question in such a diverse cohort of healthcare professionals from different ethnic, cultural and professional backgrounds.

These data highlight that most participants declared they predominantly trained using educational materials featuring light skin tones. Although Latin America and Africa used more representative resources, there were still a large percentage of respondents from these areas who reported training resources representing only white skin. These data are, unfortunately, in line with other published data. Of 1,381 images assessed in the New England of Medicine (4), 80\% of images depicted white skin and $18 \%$ depicted non-white skin $(p<0.0001)$, reporting also considerable heterogeneity in the percentage of non-white medical images published from different geographic regions and specialties (ranging from 0 to $67 \%$ ). The same group published a study in 2019 (5) which assessed diversity in published skin images in the New England Journal of Medicine and others from 1992 and 2017. Of 24,209 color photographs and 1,671 color graphics, only $22 \%$ of photographs were of darker skin tones. Importantly, they did report an increase in the use of darker skin tone images over time $(r=0.086, p<0.001)$ and an association of darker skin tone images with international authors $(r=0.12, p<0.001)$. A similar underrepresentation of darker skin tone was reported in training textbooks in Canada and US, respectively $(2,3)$. Furthermore, in two studies assessing images in plastic surgery, only $20 \%$ of images represented darker skin tones $(6,7)$. Recently, another study showed that darker skin phenotypes are underrepresented in the UWorld Step 2 QBank, a popular study tool for medical students seeking US residencies (15).

An important finding of this study is the self-reported confidence and the factors affecting it. In general, only $5 \%$ of respondents felt confident across a range of skin tones with $43 \%$ being generally uncertain if correct. As expected, working experience significantly affected self-confidence $(p<0.001)$. However, a more interesting finding was represented by the link between type of training resources and self-confidence. Only $3 \%$ of those using training resources with only whiteskin images felt confident in diagnosing skin conditions on different skin tones $(p<0.001)$. The use of more inclusive resources positively correlated with increased confidence especially when taking into account the participant's level of experience (Figure 1).

These data also highlighted that medical specialty affected reported self-confidence. Whilst the finding that dermatologists felt most confident was expected (none reported to be 
usually uncertain), both pediatricians and emergency physicians reported being generally uncertain (42 and 56\%, respectively). Data analysis according to years of experience, in the group " $6-10$ years", being an emergency physician was significantly associated with less self-confidence $(p=0.017)$. These findings need further validation but, if confirmed, will need a review of training programs and a development of more inclusive resources. This is particularly relevant since pediatricians and emergency physicians interact with a wide range of different ethnicities in emergency departments. However, although our findings are new for the pediatric field, dermatologists have already declared in previous studies that they did not feel their education had adequately prepared them to diagnose and manage skin disease in Black patients (16). Also, a recent study involving medical students showed that they were less accurate in diagnosing squamous cell carcinoma, atopic dermatitis, and urticaria in patients with skin of color (17).

Our study has some limitations. Firstly, although the platform is very-well established in the pediatric field with a wide international audience, the survey may have had a non-homogenous distribution, which could have affected participation from LMICs. In fact, participants from Latin America and Africa, and those with non-white ethnicity, were poorly represented. Secondly, health care providers with an interest in dermatology and diagnosis might have been more likely to participate and, therefore, could represent a selection bias. However, our survey still reports the largest data available on this topic globally and it seems improbable that a larger response would significantly reverse the trends. Thirdly, participants may have reported their self-confidence with a "pediatriclens", reporting their skills in recognizing only pediatric skin conditions. This may have affected the emergency physicians' self-confidence compared to their confidence in diagnosing adult skin conditions. We also acknowledge the known limitations of self-reporting of confidence and hope this study will represent the preliminary step for a future prospective study assessing ability in diagnosing skin conditions across different skin tones in an objective manner. In this regard, using English as the sole language can be a further limitation, however the questions were relatively simple. Lastly, another important limitation of this study is the self-assessment of diversity of medical resources, for a number of reasons: we could not directly address the skin representation in training resources used; also, the concept of diversity is subjective and our investigation relies on an implied subjective judgment about what counts as enough diversity. Nevertheless, despite these limitations, our findings are in line with recent positions from major societies, highlighting the urgent need of addressing equality and diversity in medical education. A recent viewpoint from US dermatologists called for a dermatologic curricula that incorporate concepts of antiracism, implicit bias, cultural humility, health disparities, and skin-ofcolor education into journal clubs, grand rounds presentations, resident lectures, and direct patient exposure (18-20) and similar devices have been raised by the American Academy of Pediatrics (21) and by European paediatricians (22).

In conclusion, our study provides preliminary data that training resources used by healthcare professionals on a global scale may lack enough diversity in representation of skin images. Importantly, our findings also provide preliminary evidence that this lack of diversity can possibly affect self-confidence in diagnosing skin conditions on a variety of skin tones, which has the potential to impact child health outcomes from correct and timely diagnosis of skin conditions on children and young people. These data need urgent prospective validation using more rigorous methodology, in order to provide evidence to redefine training programs of healthcare professionals worldwide.

\section{DATA AVAILABILITY STATEMENT}

The raw data supporting the conclusions of this article will be made available by the authors, upon request without undue reservation.

\section{ETHICS STATEMENT}

Ethical review and approval was not required for the study on human participants in accordance with the local legislation and institutional requirements. Written informed consent from the participants' legal guardian/next of kin was not required to participate in this study in accordance with the national legislation and the institutional requirements.

\section{DON'T FORGET THE BUBBLES}

The DFTB Skin Deep core team: Nikki Abela, Michelle Alisio, Safeena Afzal, Oke Obiuwevbi, Nensi Parekh, Alyah Seif, Keir Shiels, Aarani Somaskanthan, Kaylita Chantiluke, Michelle Arora, Holly Wakefield, and Rebecca Platt.

\section{AUTHOR CONTRIBUTIONS}

DB, DS, TD, and DR designed the study. J-FL and DS analyzed the data. DB drafted the manuscript which was reviewed and edited by DS, J-FL, TD, and DR. All authors contributed to the article and approved the submitted version.

\section{FUNDING}

This work was supported by Surf4Children, a no profit organization is leading a health project in Sierra Leone and was involved in images collection and supported the open access fee, without affecting the study design.

\section{ACKNOWLEDGMENTS}

We would like to thank all the participants of the survey and all members from all countries contributing to the growing community of the Skin Deep project.

\section{SUPPLEMENTARY MATERIAL}

The Supplementary Material for this article can be found online at: https://www.frontiersin.org/articles/10.3389/fped. 2022.837552/full\#supplementary-material 


\section{REFERENCES}

1. Trent M, Dooley DG, Dougé J. Section on adolescent health council on community pediatrics, committee on adolescence. The impact of racism on child and adolescent health. Pediatrics. (2019) 144:e20191765. doi: 10.1542/9781610024310-part06-ch22

2. Louie P, Wilkes R. Representations of race and skin tone in medical textbook imagery. Soc Sci Med. (2018) 202:38-42. doi: 10.1016/j.socscimed.2018.02.023

3. Lester JC, Taylor SC, Chren MM. Under-representation of skin of colour in dermatology images: not just an educational issue. Br J Dermatol. (2019) 180:1521-2. doi: 10.1111/bjd.17608

4. Massie JP, Cho DY, Kneib CJ, Sousa JD, Morrison SD, Friedrich JB. A picture of modern medicine: race and visual representation in medical literature. $J$ Natl Med Assoc. (2021) 113:88-94. doi: 10.1016/j.jnma.2020.07.013

5. Massie JP, Cho DY, Kneib CJ, Burns JR, Crowe CS, Lane M, et al. Patient representation in medical literature: are we appropriately depicting diversity? Plast Reconstr Surg Glob Open. (2019) 7:e2563. doi: 10.1097/GOX.0000000000002563

6. Cho DY, Kneib CJ, Massie JP, Sobol DL, Crowe CS, Shakir A, et al. Visual representation of racial diversity in aesthetic surgery literature. J Plast Reconstr Aesthet Surg. (2021) 74:223-43. doi: 10.1016/j.bjps.2020.05.048

7. Cho DY, Kneib CJ, Shakir A, Burns JR, Lane M, Massie JP, et al. Underrepresentation of racial minorities in breast surgery literature: a call for increased diversity and inclusion. Ann Surg. (2021) 273:202-7. doi: 10.1097/SLA.0000000000004481

8. Buonsenso D, Sollena P, Eke Tchangou M, De Rose C, Turriziani Colonna A, Sinatti D, et al. Black Kids Matter initiative calls for greater inclusion of Black children in clinical trials. Acta Paediatr. (2021) 110:19612. doi: 10.1111/apa.15741

9. The Lancet Diabetes Endocrinology. COVID-19 and Racisma double edged dagger. Lancet Diabetes Endocrinol. (2020) 8:649. doi: 10.1016/S2213-8587(20)30243-6

10. Lester JC, Jia JL, Zhang L, Okoye GA, Linos E. Absence of images of skin of colour in publications of COVID-19 skin manifestations. $\mathrm{Br}$ J Dermatol. (2020) 183:593-5. doi: 10.1111/bjd.19258

11. Gorbatenko-Roth K, Prose N, Kundu RV, Patterson S. Assessment of black patients' perception of their dermatology care. JAMA Dermatol. (2019) 155:1129-34. doi: 10.1001/jamadermatol.2019.2063

12. Jahn HK, Jahn IHJ, Roland D, Behringer W, Lyttle M. Paediatric emergency research in the United Kingdom, Ireland (PERUKI). Prescribing in a paediatric emergency: a PERUKI survey of prescribing and resuscitation aids. Acta Paediatr. (2020) 110:1038-45. doi: 10.1111/apa.15551

13. Thoma B, Goerzen S, Horeczko T, Roland D, Tagg A, Chan T, et al. An international, interprofessional investigation of the self-reported podcast listening habits of emergency clinicians: a METRIQ study. CJEM. (2020) 22:112-7. doi: 10.1017/cem.2019.427

14. Kass G. An exploratory technique for investigating large quantities of categorical data. J R Stat Soc C (Appl Stat). (1980) 29:119-27. doi: $10.2307 / 2986296$
15. Cerdeña JP, Jaswaney R, Plaisime MV, Braun L. Assessment of skin phenotype representation in a popular medical licensing educational resource. JAMA Netw Open. (2021) 4:e2033164. doi: 10.1001/jamanetworkopen.2020.3 3164

16. Buster KJ, Stevens EI, Elmets CA. Dermatologic health disparities. Dermatol Clin. (2012) 30:53-9. doi: 10.1016/j.det.2011.08.002

17. Fenton A, Elliott E, Shahbandi A, Ezenwa E, Morris C, McLawhorn J, et al. Medical students' ability to diagnose common dermatologic conditions in skin of color. J Am Acad Dermatol. (2020) 83:9578. doi: 10.1016/j.jaad.2019.12.078

18. Lester JC, Taylor SC. Resisting racism in dermatology: a call to action. JAMA Dermatol. (2021) 157:267-8. doi: 10.1001/jamadermatol.2020. 5029

19. Desai SR, Khanna R, Glass D, Alam M, Barrio V, French LE, et al. Embracing diversity in dermatology: Creation of a culture of equity and inclusion in dermatology. Int J Womens Dermatol. (2021) 7:37882. doi: 10.1016/j.ijwd.2021.08.002

20. Chang MJ, Lipner SR. Analysis of skin color in the American Academy of Dermatology basic dermatology curriculum. Int J Womens Dermatol. (2021) 7:505-7. doi: 10.1016/j.ijwd.2021.03.007

21. Unaka NI, Winn A, Spinks-Franklin A, Poitevien P, Trimm F, Nuncio Lujano BJ, et al. An entrustable professional activity addressing racism and pediatric health inequities. Pediatrics. (2022) 10:e2021054604. doi: 10.1542/peds.2021-054604

22. Shanmugavadivel D, Buonsenso D, Chantiluke K, Arora M. Importance and impact of diversity in paediatric medical education. Arch Dis Child Educ Pract Ed. (2021) 6:edpract-2020-320796. doi: 10.1136/archdischild-2020-320796

Conflict of Interest: TD and DR are executive committee members of Don't Forget the Bubbles.

The remaining authors declare that the research was conducted in the absence of any commercial or financial relationships that could be construed as a potential conflict of interest.

Publisher's Note: All claims expressed in this article are solely those of the authors and do not necessarily represent those of their affiliated organizations, or those of the publisher, the editors and the reviewers. Any product that may be evaluated in this article, or claim that may be made by its manufacturer, is not guaranteed or endorsed by the publisher.

Copyright (c) 2022 Buonsenso, Liu, Shanmugavadivel, Davis and Roland. This is an open-access article distributed under the terms of the Creative Commons Attribution License (CC BY). The use, distribution or reproduction in other forums is permitted, provided the original author(s) and the copyright owner(s) are credited and that the original publication in this journal is cited, in accordance with accepted academic practice. No use, distribution or reproduction is permitted which does not comply with these terms. 